\title{
Bilateral Adrenal Histoplasmosis in an Immunocompetent Man
}

Rukhsana Parvin* and Rafique Uddin AKM

Department of Medicine, Enam Medical College and Hospital, Savar, Dhaka, Bangladesh

\begin{abstract}
Histoplasmosis is a deep fungal infection caused by Histoplasma capsulatum. It presents commonly with transient pulmonary infection but disseminated form can involve any system in the body. Adrenal gland is affected frequently in histoplasmosis but bilateral involvement is unusual. Adrenal histoplasmosis is also common in immunocompromised host. We report a case of bilateral adrenal histoplasmosis in an immunocompetent patient without any features of adrenal insufficiency. Histoplasmosis was diagnosed by fine needle aspiration cytology (FNAC) from adrenal gland. Patient was treated with itraconazole with good response and is currently under follow-up.
\end{abstract}

Keywords: Histoplasmosis; Adrenal gland; Immunocompetent host

\section{Introduction}

Histoplasmosis is a fungal infection caused by a dimorphic saprophytic fungus Histoplasma capsulatum [1]. It is endemic in various part of the world especially in North and Latin America, south of Sahara and sporadic in Africa and Far-East Asia [2-5]. It is found as a mycelium form in the soil mixed with bat and bird droppings. Infection results from inhalation of the spores. In humans and animals, spores germinate into yeast form and accumulate in the macrophages. Histoplasma causes a spectrum of illness from subclinical infection to progressive disseminated disease. Though it affects immunocompromised people mostly, there are several reports of infections in immunocompetent persons [6,7]. Histoplasmosis involving bilateral adrenal glands as a primary infection is an unusual clinical form. A high index of suspicion is required for the diagnosis. We report a case of bilateral adrenal mass in an immunocompetent patient who presented with non-specific symptoms and responded well to antifungal treatment.

\section{Case Report}

A 32-year-old male cultivator presented with low grade intermittent fever, weight loss and anorexia for one and a half years. He lost around $10 \mathrm{~kg}$ body weight during his period. He was diagnosed as a case of tuberculosis on the basis of his clinical features and few investigation reports about fourteen months back and antitubercular treatment was started, but the patient discontinued treatment as there was no improvement. Later on, he was prescribed several antibiotics by different physicians without any success. Subsequently, he came to Enam Medical College and Hospital six months ago. On examination, he was ill-looking, mildly pale and cachectic with weight of $50 \mathrm{~kg}$ and height of $160 \mathrm{~cm}$. There was no postural drop. Examination of abdomen revealed mild hepatosplenomegaly. All other systems had normal findings. Hematological profile showed ESR $80 \mathrm{~mm}$ in $1^{\text {st }}$ hour, hemoglobin $11 \mathrm{~g} / \mathrm{dl}$, white blood cells $5700 / \mathrm{mm}^{3}$ (neutrophils $69 \%$, lymphocytes $20 \%$ and monocytes $5 \%$ ); plasma glucose $100 \mathrm{mg} /$ $\mathrm{dl}$; albumin $3 \mathrm{~g} / \mathrm{dl}$; sodium $141 \mathrm{mEq} / \mathrm{l}$; potassium $3.9 \mathrm{mEq} / \mathrm{l}$, plasma urea of $30 \mathrm{mg} / \mathrm{dl}$ and creatinine of $1.0 \mathrm{mg} / \mathrm{dl}$. Blood culture showed no growth of any bacteria or fungi. Chest radiography, renal function tests, liver function tests and bone marrow findings were normal. The test for malarial parasites, tuberculosis and HIV were negative. The adrenal endocrine profile was normal. Ultrasonography of abdomen showed bilateral adrenal mass of about $6.2 \times 4.5 \mathrm{~cm}$ and $8.6 \times 4.3 \mathrm{~cm}$ in both adrenal regions with mild hepatosplenomegaly. Contrast enhanced CT revealed bilateral, almost symmetrical, hypoattenuated adrenal glands with peripheral rim enhancement (Figure 1). Ultrasonography guided FNAC from adrenal gland was done and sent for histopathology. Gomori's Methenamine Silver (GMS) stain showed much intracellular, yeast like structures within macrophages morphologically consistent with Histoplasma capsulatum (Figure 2).

\section{Discussion}

Histoplasmosis remains asymptomatic in most of the cases. It may be divided into the following types: primary pulmonary histoplasmosis, progressive disseminated histoplasmosis, primary cutaneous histoplasmosis and african histoplasmosis [8]. Progressive disseminated disease is rare and usually seen in endemic areas. Patients with abnormal host defense mechanisms are prone to develop progressive infections. Dissemination occurs in $70 \%$ of asymptomatic cases to lymph nodes, liver, spleen, kidney, lungs and less often to adrenal gland, brain, eye and skin [9].

Diagnosis of histoplasmosis is difficult as the patients present with non-specific symptoms such as fever, weight loss, malaise and anorexia. The important differential diagnoses are tuberculosis and malignancy. In this case, initially our patient received antitubercular chemotherapy

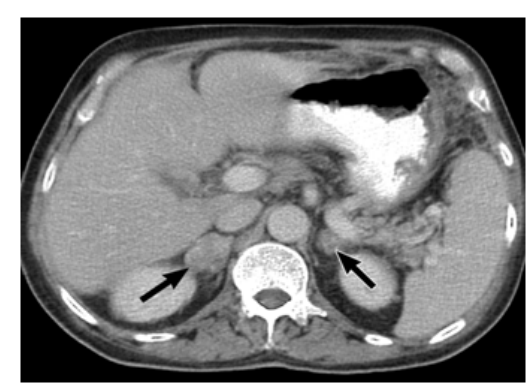

Figure 1: Contrast enhanced CT revealed bilateral hypoattenuated adrenal glands with peripheral rim enhancement.

*Corresponding author: Rukhsana Parvin, Associate Professor, Enam Medical College and Hospital, 9/3 Parbotinogor, Thana road, Savar, Dhaka, Bangladesh, Tel: 88-01815658124; E-mail: rukhsana_parvin@yahoo.com

Received February 27, 2013; Accepted May 08, 2013; Published May 13, 2013

Citation: Parvin R, Rafique Uddin AKM (2013) Bilateral Adrenal Histoplasmosis in an Immunocompetent Man. J Gen Pract 1: 103. doi: 10.4172/2329-9126.1000103

Copyright: (C) 2013 Parvin R, et al. This is an open-access article distributed under the terms of the Creative Commons Attribution License, which permits unrestricted use, distribution, and reproduction in any medium, provided the original author and source are credited. 


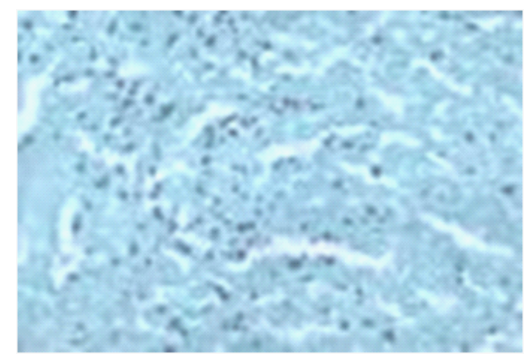

Figure 2: Yeast forms of Histoplasma capsulatum. The patient was treated with itraconazole $200 \mathrm{mg}$ twice daily as his condition was stable and clinical improvement was observed within 2 weeks. On last follow-up ( $18^{\text {th }}$ week), he was asymptomatic with increased weight of about $7 \mathrm{~kg}$

as tuberculosis is more common and prevalent in Bangladesh [10,11]. But the patient did not improve at all after five months of treatment and then he discontinued it himself. Abdominal imaging revealed bilateral adrenal enlargement with hepatosplenomegaly. The differential diagnoses of bilateral adrenomegaly are metastasis, lymphoma, adrenal hemorrhage, sarcoidosis and infections which include histoplasmosis, tuberculosis, cryptococosis, coccidioidomycosis and blastomycosis $[12,13]$. The presence of hypoattenuated adrenal glands with peripheral rim enhancement narrowed the differential diagnoses to tuberculosis and adrenal histoplasmosis. As the patient did not respond to antitubercular treatment and there were no other positive findings in favor of tuberculosis, histoplasmosis was a possible consideration. Ultrasonography guided fine needle aspiration from the right adrenal gland showed yeast like structures with GMS stain, consistent with histoplasmosis. Histoplasmosis can be further confirmed by fungal culture, polymerase chain reaction (PCR) and urine antigen tests. In our case, PCR and urine antigen tests were not done due to limited facility.

Adrenal histoplasmosis in immunocompetent host is reported in several studies internationally $[6,7,14-16]$ and nationally $[17,18]$. In this case, the patient had no features of immunocompromisation. $\mathrm{He}$ was a cultivator and this could have been a risk factor as soil is the natural inhabitant of histoplasma spores.

Adrenal insufficiency due to adrenal histoplasmosis is not very common. In this case, the patient did not have any features of adrenal insufficiency. Kauffman [19] found adrenal involvement in only 12 out of 58 patients with histoplasmosis and none of them showed clinical evidence of adrenal failure [19]. Similar observations are found in other studies $[14,20]$.

Amphotericin B is the recommended treatment for critically ill patient whereas oral itraconazole is the choice of drug in stable patient. Among patients without AIDS, amphotericin B is effective in $68-72 \%$ of the cases and itraconazole is effective in $100 \%$ of the cases [21]. The response rate is good though there is chance of relapse if treatment is discontinued. So it should be continued for one to two years [22]. Our patient is still under treatment with good response and is on regular follow-up.

Adrenal histoplasmosis should be considered in any patient presenting with unilateral or bilateral adrenal mass with constitutional symptoms especially in endemic areas. Early diagnosis and treatment can save the patient from serious complications.

\section{References}

1. Bregani ER, Van Tien T, Ceraldi T, Delfitto C, Figini G (2000) Histoplasmosis: not only a tropical disease. Recenti Prog Med 91: 396-401.
2. Wheat LJ (2006) Histoplasmosis: a review for clinicians from non-endemic areas. Mycoses 49: 274-282.

3. Chu JH, Feudtner C, Heydon K, Walsh TJ, Zaoutis TE (2006) Hospitalizations for endemic mycoses: a population-based national study. Clin Infect Dis 42 : 822-825.

4. Goodwin RA Jr, Shapiro JL, Thurman GH, Thurman SS, Des Prez RM (1980) Disseminated histoplasmosis: clinical and pathologic correlations. Medicine (Baltimore) 59: 1-33.

5. Adderson E (2006) Histoplasmosis. Pediatr Infect Dis J 25: 73-74.

6. Benevides CF, Durães RO, Aquino B, Schiavon Lde L, Narciso-Schiavon JL, et al. (2007) Bilateral adrenal histoplasmosis in an immunocompetent man. Rev Soc Bras Med Trop 40: 230-233.

7. Rana C, Krishnani N, Kumari N (2011) Bilateral adrenal histoplasmosis in immunocompetent patients. Diagn Cytopathol 39: 294-296.

8. William DJ (2006) Andrews' Diseases of the Skin: Clinical Dermatology. (9thedn), W.B. Saunders Company, Philadelphia, USA.

9. Gibb WR, Ramsay AD, McNeil NI, Wrong OM (1985) Bilateral adrenal masses Br Med J (Clin Res Ed) 291: 203-204.

10. Zaman K, Yunus M, Arifeen SE, Baqui AH, Sack DA, et al. (2006) Prevalence of sputum smear-positive tuberculosis in a rural area in Bangladesh. Epidemiol Infect 134: 1052-1059.

11. Zaman K, Hossain S, Banu S, Quaiyum MA, Barua PC, et al. (2012) Prevalence of smear-positive tuberculosis in persons aged $\geq 15$ years in Bangladesh: results from a national survey, 2007-2009. Epidemiol Infect 140: 1018-1027.

12. Schonfeld AD, Jackson JA, Smith DJ, Hurley DL (1991) Disseminated histoplasmosis with bilateral adrenal enlargement: diagnosis by computed tomography-directed needle biopsy. Tex Med 87: 88-90.

13. Rozenblit AM, Kim A, Tuvia J, Wenig BM (2001) Adrenal histoplasmosis manifested as Addison's disease: unusual CT features with magnetic resonance imaging correlation. Clin Radiol 56: 682-684.

14. Kumar N, Singh S, Govil S (2003) Adrenal histoplasmosis: clinical presentation and imaging features in nine cases. Abdom Imaging 28: 703-708.

15. Desmet P, Vogelaers D, Afschrift M (2004) Progressive disseminated histoplasmosis 10 years after return out of Africa in an immunocompetent host. Acta Clin Belg 59: 274-278.

16. Mahajan R, Sharma U, Trivedi N, Prasad M, Kansra U, et al. (2000) Histoplasma capsulatum in adrenal gland aspirate--a case report. Indian J Pathol Microbiol 43: 165-168.

17. Amin RM, Shumi F, Khan H, Abdullah SA, Alam S, et al. (2011) Disseminated histoplasmosis in an elderly man presented with fever, weight loss, abdominal pain and haemoptysis-a case report with literature review. J Medicine 2011: 81-85

18. Alam ABM, Hasan Z, Khan MAI, Zulkifl MA, Samdani ATM, et al. (2011) Chronic disseminated histoplasmosis in an immunocompetent man presented as bilateral adrenal masses with partial adrenocortical insufficiency - a rare condition. Journal of Bangladesh College of Physicians and Surgeons 29: 235240.

19. Kauffman CA (2001) Fungal infections in older adults. Clin Infect Dis 33: 550555

20. Goswami RP, Pramanik N, Banerjee D, Raza MM, Guha SK, et al. (1999) Histoplasmosis in eastern India: the tip of the iceberg? Trans R Soc Trop Med Hyg 93: 540-542.

21. http://dx.doi.org/10.1590/S1806-37132009001100013

22. Johnston AW, Brown PA, Ewen SW (1996) Histoplasmosis--a ten year followup. J Infect 33: 111-113. 